\title{
An Algebraic Proof of the Existence of a Competitive Equilibrium in Exchange Economies
}

\author{
Guang-Zhen Sun \\ Department of Economics, Faculty of Social Sciences, University of Macau, Macau, China \\ Email: gzsun@umac.mo
}

Received 13 November 2013; revised 13 December 2013; accepted 30 December 2013

Copyright (C) 2014 by author and Scientific Research Publishing Inc.

This work is licensed under the Creative Commons Attribution International License (CC BY). http://creativecommons.org/licenses/by/4.0/

c) (i) Open Access

\begin{abstract}
The standard excess demand argument for existence of competitive equilibria in exchange economies invokes maximizing the market value of the aggregate excess demand function and thereby adjusting the prices toward equilibrium. By exploiting the Perron-Frobenius theorem on stochastic matrices, we offer an algebraic proof of the existence of a competitive equilibrium without resorting to such a device of excess demand.
\end{abstract}

\section{Keywords}

Exchange Economy, Existence of Competitive Equilibrium, Perron-Frobenius Theorem

\section{Introduction}

The standard excess demand argument for existence of competitive equilibriain exchange economies invokes maximizing the value of the excess demand of the economy (the aggregate excess demand) and thereby adjusting the prices toward equilibrium [1]-[3]. At least for pedagogical purpose, one may legitimately interpret the adjustment procedure, often referred to as tatonnement, as that in which a price-setting agency, the so-called Walrasian auctioneer, gathers the information as regards to each agent's excess demand and then fine-tunes the prices so as to raise the prices of the commodities that are over-demanded (with a positive aggregate excess demand registered) and to lower the prices of the commodities that are under-demanded (with a negative aggregate excess demand registered). It is true that the excess demand function is a very useful device that renders the fixed point theory nicely applicable to the proof of the existence of a market-clear price vector. Such a technical device, powerful though as it is in remarkably simplifying the formulation, nonetheless suggests a centralized coordination mechanism. Exploiting the Perron-Frobenius theorem on stochastic matrices, a well known result 
in linear algebra, we provide an alternative proof of the existence of a competitive equilibrium without resorting to such an artificial price-setting mechanism. It is worth pointing out that in so doing our argument does not invoke aggregating the individuals' excess demand, let alone to maximizing the market value of the aggregate excess demand. To the extent that the price-setting agency, as is embodied by the Walrasian auctioneer, personifies the coordination of the decentralized price system, "the invisible hand", and hence rendering it more or less visible, our approach appears to be conceptually more natural. But a cost of awkwardness in algebraic manipulation has to be paid in our undertaking, compared to the rather neat formulation based on the Walrasian tatonnement. However, such a cost may be well justifiable for an economically appealing argument about the important idea of the invisible hand.

\section{The Proof}

We first restate the classical result on the existence of a competitive equilibrium.

Theorem. For any pure exchange economy with $n$ commodities and $m$ agents, in which each agent $\tau \in\{1, \cdots, m\}$ has a preference represented by a utility function $U^{\tau}: R_{+}^{n} \rightarrow R$ that is continuous, concave and strongly monotone and an endowment $\omega^{\tau} \in R_{+}^{n}$ such that the endowment of any commodity for the economy as a whole is positive, i.e., $\sum_{\tau=1}^{m} \omega^{\tau} \gg 0$, there exists a competitive equilibrium.

Proof. First of all, normalize the endowment of each commodity of the community as one, i.e., $\sum_{\tau=1}^{m} \omega_{i}^{\tau}=1$, $\forall i \in\{1, \cdots, n\}^{1}$. Consider the price simplex $S_{n} \equiv\left\{p \mid p_{i} \geq 0, \forall i\right.$ and $\left.\sum_{i=1}^{n} p_{i}=1\right\}$. For any interior point $p$, let $Y^{\tau}(p) \equiv\left\{\left[\frac{p_{1} x_{1}^{\tau}(p)}{\omega^{\tau} \cdot p}, \cdots, \frac{p_{n} x_{n}^{\tau}(p)}{\omega^{\tau} \cdot p}\right]^{\mathrm{T}} \mid x^{\tau}(p) \in \arg \max _{p x \leq p \omega^{\tau}, x \geq o} U^{\tau}(x)\right\}, \tau=1, \cdots, m$. Notice that for any

$y^{\tau} \in Y^{\tau}(p)$ its i-th component is the percentage share of agent $\tau$ 's wealth spent on commodity $i$. Monotone preference implies $\sum_{i=1}^{n} y_{i}^{\tau}=1$. Convexity of preferences implies that for any agent $\tau, Y^{\tau}(p)$ is convex at any interior point of $S_{n}$. Also note that $Y^{\tau}(p)$ is upper-semi-continuous by Berge's maximum theorem. For any $p \in \partial S_{n}$, let $Y^{\tau}(p)$ be the convex hull of the limit points of any possible $\left\{y^{k}, y^{k} \in Y^{\tau}\left(p^{k}\right)\right\}$ where the sequence of interior points $\left\{p^{k}\right\}$ approaches $p$. That is, $Y^{\tau}(p) \equiv \operatorname{Co}\left\{y \mid \exists\left\{p^{k}\right\}\right.$ and $\left\{y^{k}\right\}$ such that $\forall k, p^{k} \in S_{n} \backslash \partial S_{n}, y^{k} \in Y^{\tau}\left(p^{k}\right)$ and that $p^{k} \rightarrow p, y^{k} \rightarrow y$ as $\left.k \rightarrow \infty\right\}$. By definition, $Y^{\tau}(p)$ is upper-semi-continuous and convex-valued at any $p \in \partial S_{n}{ }^{2}$.

Let $F\left(p, y^{1}(p), \cdots, y^{m}(p)\right) \equiv\left[\sum_{\tau=1}^{m} y_{i}^{\tau}(p) \omega_{j}^{\tau}\right]_{n \times n}$ The sum of each column of $F$ equals $\sum_{i=1}^{n} \sum_{\tau=1}^{m} y_{i}^{\tau} \omega_{j}^{\tau}=\sum_{\tau=1}^{m}\left(\sum_{i=1}^{n} y_{i}^{\tau}\right) \omega_{j}^{\tau}=\sum_{\tau=1}^{m} \omega_{j}^{\tau}=1$, hence $F$ is a column stochastic matrix. Consequently, the Perron-Frobenius eigenvalue of $F$ is one and there exists at least one corresponding eigenvector $q \in S_{n}$, i.e., $F q$

${ }^{1}$ One may do so by considering $\left(w_{i}^{\tau}\right)^{\prime} \equiv w_{i}^{\tau} /\left(\sum_{s=1}^{m} w_{i}^{s}\right)$ for any agent $\tau$ and commodity $i$ and the prices adjusted to the accordingly changed units used to measure each commodity.

${ }^{2} \mathrm{~A}$ careful reader might be concerned with the closedness of the correspondence graph along $\partial S_{n}$. But it is not a problem. Consider any $\left\{p^{k}\right\} \subset \partial S_{n}$ and $\left\{y^{k}\right\}$ such that $\forall k, y^{k} \in Y^{\tau}\left(p^{k}\right)$ and $p^{k} \rightarrow p$ as $k \rightarrow \infty$. One needs to come to grips with two scenarios. First of all suppose that for any $k, \quad y^{k}$ is a limit when some sequence of strictly positive price vectors approaches $p$. By the definition of $Y^{\tau}$ on $\partial S_{n}$, for any $\mathrm{k}$ there exists $\left\{\left(\tilde{p}^{k}\right)^{s}\right\}_{s \geq 1} \subset S_{n} \backslash \partial S_{n}$ and $\left(\tilde{y}^{k}\right)^{s} \in Y^{\tau}\left(\left(\tilde{p}^{k}\right)^{s}\right), \forall s$, such that $\left(\tilde{p}^{k}\right)^{s} \rightarrow p^{k}, \quad\left(\tilde{y}^{k}\right)^{s} \rightarrow y^{k} \in Y^{\tau}\left(p^{k}\right)$ as $s \rightarrow \infty$. One can thus appropriately construct a sequence $\left\{p^{t}\right\}_{t 21} \subset S_{n} \backslash \partial S_{n}$, with $p^{t} \in\left\{\left(\tilde{p}^{t}\right)^{s}\right\}_{s>1}$, which converges to $p$ and to which a sequence of $\left\{y^{t}\right\}_{t \geq 1}$, converging to $y \in Y^{\tau}(p)$, is associated. Similar argument yet with more awkward notations applies to another scenario in which for some or all $k, y^{k}$ is not a limit point but a convex combinations of two vectors each of which is a limit point when one sequence of strictly positive price vectors approaches $p$. 
$=q[4]$.

Consider any sequences $\left\{p^{k}\right\}$ and $\left\{q^{k}\right\}$ such that there exist $\left(y^{\tau}\right)^{k} \in Y^{\tau}\left(p^{k}\right), \tau=1, \cdots, m$, satisfying $F^{k} p^{k}=q^{k}$ for any $k$ where $F^{k} \equiv F\left(p^{k},\left(y^{1}\right)^{k}, \cdots,\left(y^{m}\right)^{k}\right)$ and that $p^{k} \rightarrow p^{*}, q^{k} \rightarrow q^{*}$ as $k \rightarrow \infty$. WLOG, assume $\forall \tau,\left(y^{\tau}\right)^{k} \rightarrow y^{*^{\tau} \tau}$. As is established in the above, $Y^{\tau}(p)$ is upper-semi-continuous. Hence, $y^{* \tau} \in Y^{\tau}\left(p^{*}\right)$. Take the limit on both sides of $F^{k} p^{k}=q^{k}$ as $k \rightarrow \infty, F^{*} p^{*}=q^{*}$ where $F^{*} \equiv F\left(p^{*}, y^{* 1}, \cdots, y^{* m}\right)$. The correspondence from $\mathrm{p}$ to $q$ is closed.

We now prove that $\forall p \in S_{n},\{q(p)\}$ is convex. Consider any two elements in $\{q(p)\}$ say $q^{A}$ and $q^{B}$, i.e., there exist $y^{A_{\tau}} y^{B_{\tau}} \in Y^{\tau}(p), \quad \tau \in\{1, \cdots, m\}$ such that $\left[y^{A_{1}} \cdots y^{A_{m}}\right] W q^{A}=q^{A}$ and $\left[y^{B_{1}} \cdots y^{B_{m}}\right] W q^{B}=q^{B}$ where $W \equiv\left[\omega_{j}^{\tau}\right]_{m \times n}$. For any $\beta \in[0,1]$, we claim that there exist $\alpha_{\tau} \in[0,1], \tau=1, \cdots, m$ such that

$$
\left[\alpha_{1} y^{A_{1}}+\left(1-\alpha_{1}\right) y^{B_{1}}+\cdots+\alpha_{m} y^{A_{m}}+\left(1-\alpha_{m}\right) y^{B_{m}}\right] W\left(\beta q^{A}+(1-\beta) q^{B}\right)=\beta q^{A}+(1-\beta) q^{B}
$$

Note the term in the brackets of LHS of Equation (1) equals

$$
\alpha_{1}\left[y^{A_{1}} 0_{n} \cdots 0_{n}\right]+\left(1-\alpha_{1}\right)\left[y^{B_{1}} 0_{n} \cdots 0_{n}\right]+\cdots+\alpha_{m}\left[0_{n} \cdots 0_{n} y^{A_{m}}\right]+\left(1-\alpha_{m}\right)\left[0_{n} \cdots 0_{n} y^{B_{m}}\right]
$$

Also notice that the RHS of Equation (1) equals

$$
\left\{\left[y^{A_{1}} 0_{n} \cdots 0_{n}\right]+\cdots+\left[0_{n} \cdots 0_{n} y^{A_{m}}\right]\right\} W \beta q^{A}+\left\{\left[y^{B_{1}} 0_{n} \cdots 0_{n}\right]+\cdots+\left[0_{n} \cdots 0_{n} y^{B_{m}}\right]\right\} W(1-\beta) q^{B}
$$

Substituting the above and (2) into (1) yields,

$$
\forall s=1,2, \ldots, n, \sum_{\tau=1}^{m}\left[y_{s}^{B_{\tau}}-y_{s}^{A_{\tau}}\right]\left[\left(1-\alpha_{\tau}\right) \beta \sum_{i=1}^{n} \omega_{i}^{\tau} q_{i}^{A}-\alpha_{\tau}(1-\beta) \sum_{i=1}^{n} \omega_{i}^{\tau} q_{i}^{B}\right]=0
$$

Since $q^{A}, q^{B} \in S_{n}$ and $\omega^{\tau} \in[0,1]^{n}, \forall \tau$, there apparently exists $\alpha_{\tau} \in[0,1], \tau=1, \cdots, m$, such that $\left(1-\alpha_{\tau}\right) \beta \sum_{i=1}^{n} \omega_{i}^{\tau} q_{i}^{A}-\alpha_{\tau}(1-\beta) \sum_{i=1}^{n} \omega_{i}^{\tau} q_{i}^{B}$, i.e., (1) holds. That is, for any $\in S_{n},\{q(p)\}$ is a convex set.

By Kakutani's theorem, there exists $p^{*} \in S_{n}$, such that $F\left(p^{*}, y^{1}\left(p^{*}\right), \cdots, y^{m}\left(p^{*}\right)\right) p^{*}=p^{*}$ where $y^{\tau}\left(p^{*}\right) \in Y^{\tau}\left(p^{*}\right), \tau=1, \cdots, m$. We now show that $p^{*} \notin \partial S_{n}$. For otherwise, then by the definition of $Y^{\tau}(p)$ on $\partial S_{n}$, one possibility is that there exist sequences $\left\{p^{k}\right\}$ and $\left\{\left(y^{\tau}\right)^{k}\right\}$ such that $\forall k, p^{k} \in S_{n} \backslash \partial S_{n},\left(y^{\tau}\right)^{k} \in Y^{\tau}\left(p^{k}\right)$ and that $p^{k} \rightarrow p^{*} \in \partial S_{n},\left(y^{\tau}\right)^{k} \rightarrow y^{\tau}\left(p^{*}\right)$ as $k \rightarrow \infty$, that is, $\lim _{k \rightarrow \infty}\left[\left(y^{1}\right)^{k} \cdots\left(y^{m}\right)^{k}\right] W p^{*}=p^{*}$. WLOG, assume $p_{1}^{*}>0$ but some other components of $p^{*}$ are zeros. Then we obtain from $\lim _{k \rightarrow \infty}\left[\left(y^{1}\right)^{k} \cdots\left(y^{m}\right)^{k}\right] W p^{*}=p^{*}$ that $\lim _{k \rightarrow \infty} \sum_{\tau=1}^{m}\left(y_{s}^{\tau}\right)^{k} \omega_{1}^{\tau}=0$ for any $s$ such that $p_{s}^{*}=0$.

Thus for an agent $\tau$ endowed with a positive amount of commodity 1 (existence of such agent is guaranteed by the assumption that $\left.\sum_{\tau=1}^{m} \omega_{1}^{\tau}>0\right) \lim _{k \rightarrow \infty}\left(y_{s}^{\tau}\right)^{k}=0$ for anys such that $p_{s}^{*}=0$, an impossibility in light of the strongly monotone preferences. In the case that $Y^{\tau}(p)$ itself is not a limit point for any sequence of interior price vectors that approaches $p^{*}$, but a convex combination of two such limit points, the above application applies to each of these two limit points. Thus, $p^{*} \gg 0$. By simple algebraic manipulation one obtains from $F\left(p^{*}\right) p^{*}=p^{*}$ that $\forall i, \sum_{\tau=1}^{m} x_{i}^{\tau}\left(p^{*}\right)=1=\sum_{\tau=1}^{m} \omega_{i}^{\tau}$, i.e., all markets clear.

\section{References}

[1] Debreu, G. (1983) Four Aspects of the Mathematical Theory of Economic Equilibrium. In: Mathematical Economics: Twenty Papers of Gerard Debreu, Cambridge University Press, New York, 217-231.

[2] Mas-Colell, A., Whinston, M.D. and Green, J. (1995) Microeconomic Theory. Oxford University Press, Oxford.

[3] Jehle, G.A. and Reny, P.J. (2000) Advanced Microeconomic Theory. 2nd Edition, Addison Wesley, Boston.

[4] Aldrovandi, R. (2001) Special Matrices of Mathematical Physics. World Scientific, Singapore. 